\title{
Research on optimization of signal phase at intersection based on VISSIM simulation software
}

\author{
Zou Yong \\ Chang'an university, Middle Section of Nan Erhuan Road, Xi'an City, Shannxi Province, 710064, \\ China
}

email: 281127573@qq.com

Keywords: urban traffic; signal phase optimization scheme; VISSIM simulation; road intersection; conflicts between pedestrian and vehicle; evaluation index

\begin{abstract}
In order to reduce the conflict between pedestrian and vehicle at intersection under real-time changes, different traffic status at cross intersection and T-shaped intersection two typical intersections on present urban roads are chosen as analyzing objects, and through the redesigning of signal phrases, a signal phases optimization scheme to eliminate the conflicts between pedestrian and vehicle at theses intersections is offered. For testing the possibility of the optimization scheme,a set of evaluation indexes are constructed, and the optimization scheme are simulated at two different typical intersections respectively in Hefei and Xi'an based on Vissim software. the result of simulation showed that the evaluation indexes such as points of conflict,delay of pedestrian and vehicle, park rate, time and space resources of pedestrians are substantially improved when the optimization scheme is applied at the intersections.
\end{abstract}

\section{Introduction}

The urban road intersection, as a high incidence area of traffic accidents, exists problems of severe conflicts between pedestrian and steering vehicle or larger delay between pedestrian and vehicle[1]. Existing domestic literature mainly studies the urban road intersection on the following three aspects: 1. the analysis of traffic characteristics of pedestrian crossing[2]; 2. the analysis of conflicts and safety between pedestrian and steering vehicle[3]; 3. separation measures of conflicts between pedestrian and vehicle at intersections. With respect to a research on separation measures of conflicts between pedestrian and vehicle at intersections, Ma Wanjing et al. divide an intersection into 4 quadrants by setting up a safety island for pedestrian crossing, make full use of intersection space-time resources to optimize signal phases of signal intersection under a condition of second crossing, and analyze time and space conditions used in the method[4]. After comparison, Yang Xiaoguang, Ma Wanjing et al. analyze 2 efficiency indexes of traffic capacity which respectively stand for average pedestrian delay and vehicle flow at an intersection, and on the basis of it, demonstrate conditions of special signal phase installed in the intersection for pedestrians[5]. Shao Chunfu comprehensively analyzes the timing control research on pedestrian traffic signal, respectively introduces conditions of setting up pedestrian signals, basic control parameters of pedestrian signal and major design procedures of timing evaluation indexes of pedestrian signal and pedestrian signal designs, and suggests to take account of the pedestrian safety factor and the pedestrian crossing violation factor during a process of designing pedestrian signals[6]. In above separation measures of conflicts between pedestrian and vehicle at intersections, the research of separation measures of pedestrian and vehicle time chiefly focuses on the study on signal timing, and in addition, most optimization of pedestrian crossing traffic signal control optimizes the signal timing scheme according to historical data based on a fixed scheme of phase and phase sequence, which becomes difficult to adapt traffic conditions of real-time changes at intersections. The paper puts forward main points of signal phase control optimization in accordance with optimization essence of pedestrian and vehicle time separation, and then proposes signal phase optimization measures to decrease conflicts between pedestrian and vehicle by analyzing various traffic conditions of different road intersections. In the simulation result of actual intersection, the 
optimization measure shows that the signal phase optimization measures raised by the paper can reduce conflicts between pedestrian and vehicle at intersections.

\section{Measure optimization of reducing conflicts between pedestrian and vehicle at road intersections}

The major principle of signal control optimization at intersections is exactly to minimize the number of conflict point within a signal period as short as possible, change a permission-type right turn phase to a protection-type one, and not increase delay of right turn vehicle and improve the safety factor at intersections in the meantime. The key points of signal control optimization mainly include optimization of signal phase and phase sequence and confirmation of minimum green light time of pedestrian crossing. I. The phase optimization. A symmetrical phase is mostly adopted now and its phase combination principle is to use minimum phases and avoid conflicts in traffic flows as far as possible. II. The phase sequence optimization. When assuring a phase sequence, smoothness of phases before and after connecting should be considered in order to minimize delay of vehicle and pedestrian. In general, a straight phase sequence is in front and a steering one is in the back. III. The minimum green light time of pedestrian crossing. The minimum time for pedestrians to safely cross the street is impacted by the crossing width and becomes different following difference of phase and phase sequence.Then, the cross intersection and T-shaped intersection are taken as an example to analyze.

Optimization design of cross intersection signal phase. In order to overcome deficiencies of traditional pedestrian traffic control way, a way of pedestrian crossing between vertical zebra crossing and double diagonal zebra crossing may be selected, and meanwhile a relevant signal phase scheme should be coordinated to thoroughly separate pedestrian and steering vehicle in space, namely TSC (time separate control). When conflicts between pedestrian and vehicle are totally eliminated at the same time, the delay for pedestrian and vehicle is less than that of pedestrian crossing under a way of special pedestrian signal control. The feature of this control way is exactly capable of completely eliminating conflict points, namely, there has no influence of steering vehicle during a pedestrian signal period and has no disturbance of pedestrian during a process of vehicle turning. The following optimizes signal phases of road intersection under different traffic situation just as shown in figure 1. (1) The one-way to one-way road intersection. A common one-way to one-way road intersection only has 2 phases, so there should have 3 phases in corresponding intersections when setting up pedestrian crossing in a way of single diagonal. In the phase I, north-to-south straight vehicles and pedestrians on left and right sides are allowed to pass; in the phase II, turning vehicles at each entrance and pedestrians at diagonals are allowed to pass; and in the phase III, east-west straight vehicles and pedestrians on both sides are allowed to pass. (2) The one-way and two-way intersection. There are 4 signal phases under this form of intersection as shown in figure 1. In the phase I, east-west straight vehicles and pedestrians are allowed to pass; in the phase II, only vehicles which turn right at north entrance and turn left at east entrance are allowed to pass and pedestrians in the northeast-southwest diagonal direction can pass free; in the phase III, (one-way) straight vehicles at south entrance and pedestrians at south-north zebra crossing are allowed to pass; and in the phase IV, pedestrians in all directions are forbidden to pass, all (one-way) vehicles at north entrance and right turn vehicles at west entrance are allowed to pass.

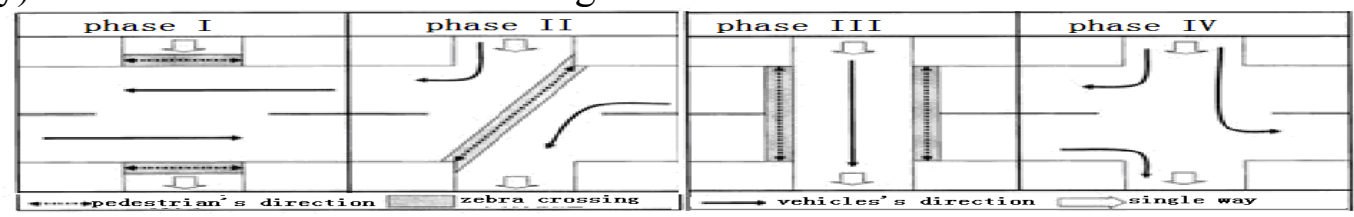

Fig.1 Signals for pedestrian at one-way to two-way intersection

Optimization design of T-shaped intersection signal phase. For the T-shaped intersection, because a permission-type right turn phase of right turn vehicle is set in most intersection signals and a permission-type phase is deemed to be the left turn vehicle phase, so pedestrians have conflicts both with right turn and left turn vehicles when crossing the street. The signal phase 
optimization aims at maximizing the safe factor of intersection and minimizing the number of conflict point. The signal phase method after improvement is shown as figure 2 and right turn improved vehicles in all directions use a protection-type right turn phase.

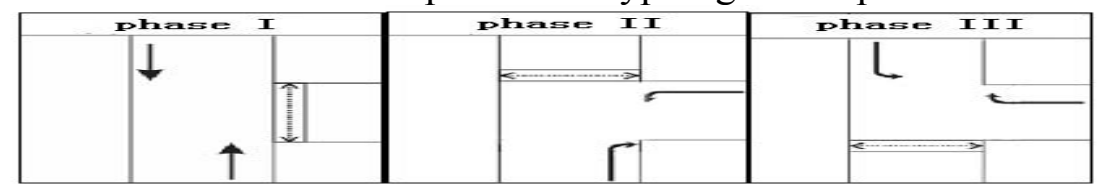

Fig. 2 Optimization signal phases at T-shaped intersection

After improvement, the conflict points of T-shaped crossing is completed eliminated and confluent points and diverging points decrease obviously, thus not only promoting the safe factor of intersection but also increasing the green light signal utilization rate in all directions of part intersections and decreasing the space-time resource waste of traffic system at intersections.

\section{Evaluation indexes of traffic system optimization at road intersections}

According to research findings at home and abroad, the signal control quality of road intersection can be measured by evaluation indexes, and it is mainly evaluated from 3 aspects- intersection safety, traffic capacity and pollution. The paper chooses delay time of pedestrian and vehicle, parking rate, frequency of conflicts between pedestrian and vehicle and pedestrian space-time resource as evaluation indexes of signal control, and then they will be introduced respectively: (1) The delay time of pedestrian and vehicle. The delay at intersections includes pedestrian crossing delay and vehicle delay and refers to difference, which is mainly caused by signal control and traffic conflicts, between actual time and ideal time when pedestrians and vehicles pass through an intersection. This index can simultaneously reflect other indexes like rapidity to pass an intersection for pedestrian and vehicle, congestion degree at intersections and oil consumption of vehicle. Therefore, the delay evaluation is the most commonly used evaluation index when evaluating an intersection signal control scheme. (2) The parking rate. It indicates the ratio of the number of parking vehicles (including complete parking and incomplete parking) to the total number of passing vehicles within a period in arriving vehicles within a signal period at an intersection. (3) The frequency of conflicts. It means the number of conflict points within each signal period. Because traffic conflicts and accidents exist an alternative, so conflicts points can be potential traffic accident points and an important index of evaluating safety levels of intersection signal control. (4) The pedestrian space-time resource. It (TS) means a sum of time and space resources provided by intersection for pedestrian crossing within each signal period.

\section{Simulation demonstration of traffic system optimization at road intersections}

At present, common traffic simulation softwares include VISSIM, PARAMICS and SYNCHRO. Because in these 3 softwares, VISSIM can have simulation demonstration to diagonal pedestrian crossing at intersections, hence, it is selected to be a tool in the analysis.

Cross intersection. The Shihe-qingyang cross intersection in Luyang District, Hefei City is taken as an example. The data of vehicle delay at each entrance: the delay time has $41.6 \mathrm{~s}$ for north entrance, $39.5 \mathrm{~s}$ for south entrance, $34.8 \mathrm{~s}$ for east entrance, and $36.2 \mathrm{~s}$ for west entrance. Its average time has $38.1 \mathrm{~s}$. According to larger quantity demanded of pedestrian crossing in the northwest-southeast direction at this intersection and situation that pedestrians need to pass traffic lights twice for crossing a street, the north-south special left turn existing in the intersection is used in coordination and the diagonal in the northwest-southeast direction for pedestrian crossing is set. The Shihe-qingyang intersection signal now adopts a three-phase signal to control and utilizes Synchrol to optimize signal timing. The signal timing scheme before and after optimization is shown as table 1: 
Table 1 Optimization signal phases at Shihe-qingyang intersection

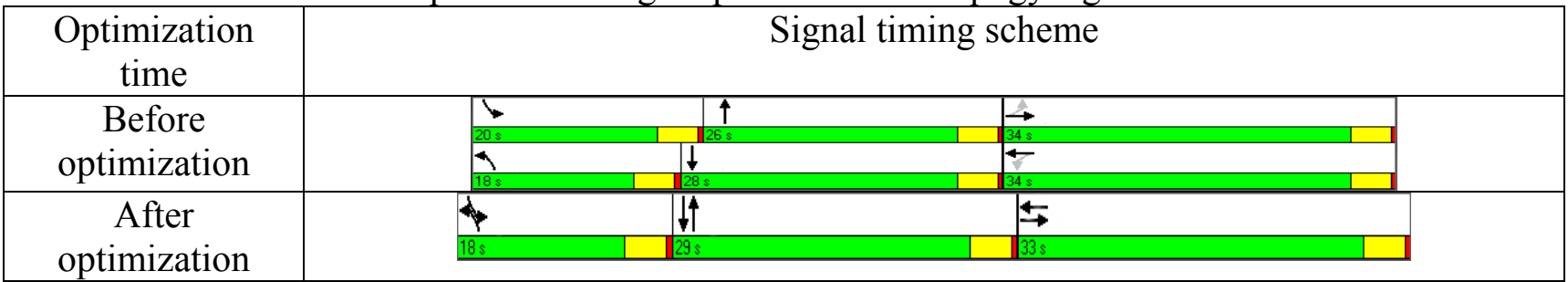

After simulating optimization measures by means of adopting the VISSIM software, result comparison analysis of status and simulation is shown as table 2:

Table 2 Comparison diagram of traffic status at Shihe-qingyang intersection

\begin{tabular}{|c|c|c|c|c|c|}
\hline $\begin{array}{c}\text { Project } \\
\text { Description }\end{array}$ & $\begin{array}{c}\text { Avehicle } \\
(\mathrm{s})\end{array}$ & $\begin{array}{c}\text { Pedestrian } \\
\text { average delay } \\
(\mathrm{s})\end{array}$ & $\begin{array}{c}\text { Parking } \\
\text { rates }(\%)\end{array}$ & $\begin{array}{c}\text { Conflict } \\
\text { Intervals }\end{array}$ & $\begin{array}{c}\text { Pedestrian space and } \\
\text { time resources } \\
\left(\mathrm{m}^{2} \cdot \mathrm{s}\right)\end{array}$ \\
\hline $\begin{array}{c}\text { Before } \\
\text { improved }\end{array}$ & 38.7 & 36.7 & 75.4 & 8 & 16128 \\
\hline After improved & 35.6 & 30.8 & 70.4 & 4 & 17452 \\
\hline
\end{tabular}

As shown in the table above, the average vehicle delay of simulations status is $38.7 \mathrm{~s}$ which has little difference from the delay of $38.1 \mathrm{~s}$ obtained by status survey, therefore, the Vissim simulation model is effective. Meanwhile, it can be seen that due to elimination of conflicts between pedestrian and vehicle after optimization, delays of vehicle and pedestrian all reduce and the vehicle's parking rate and parking frequency have a larger decrease range.

T-shaped intersection. The Cuihua-yucai T-shaped intersection in Yanta District, Xi'an City is taken as an example. Thereof, both Yucai Road and Cuihua Road are two-way four-lane roads and belong to urban secondary trunk roads. The pedestrian signal control scheme adopts a signal means of both controlling vehicles and pedestrians, lets pedestrians and straight vehicles in their direction pass simultaneously and sets the right turn phase in a permission-type right turn. Delay data of vehicle at each entrance are: the delay time has $19.2 \mathrm{~s}$ for north entrance, $25.8 \mathrm{~s}$ for south entrance and $28.5 \mathrm{~s}$ for east entrance. Its average time has $24.8 \mathrm{~s}$. In accordance with the signal phase optimization scheme raised by the paper aiming at pedestrian crossing at T-shaped intersections, the three-phase is adopted to optimize this intersection. The signal timing at Cuihua-yucai intersection status is the two-phase with a signal period of $83 \mathrm{~s}$ and uses Synchrol to optimize. The signal timing scheme before and after optimization is shown as table 3 :

Table 3 Optimization signal phases at Cuihua-yucai intersection

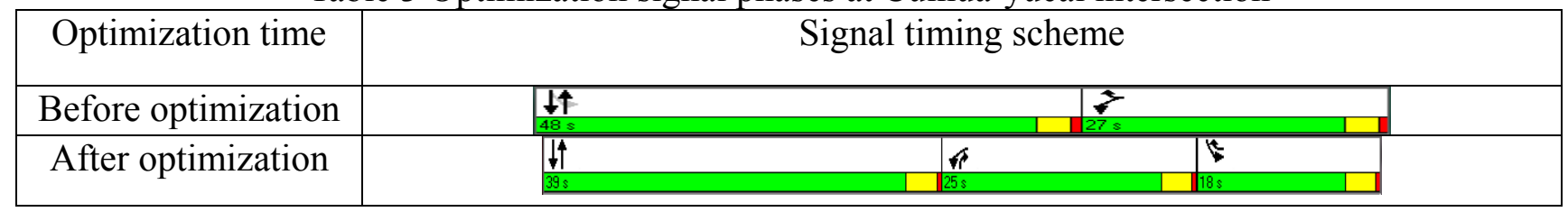

After simulating optimization measures by means of adopting the VISSIM software, result comparison analysis of status and simulation is shown as table 4:

Table 4 Comparison diagram of traffic status at Cuihua-yucai intersection

\begin{tabular}{|c|c|c|c|c|c|}
\hline $\begin{array}{c}\text { Project } \\
\text { Description }\end{array}$ & $\begin{array}{c}\text { Vehicle Average } \\
\text { delay }(\mathrm{s})\end{array}$ & $\begin{array}{c}\text { Pedestrian } \\
\text { average delay } \\
(\mathrm{s})\end{array}$ & $\begin{array}{c}\text { Parking rates } \\
(\%)\end{array}$ & $\begin{array}{c}\text { Conflict } \\
\text { points }\end{array}$ & $\begin{array}{c}\text { Pedestrian space } \\
\text { and time } \\
\text { resources } \\
\left(m^{2} \cdot s\right)\end{array}$ \\
\hline $\begin{array}{c}\text { Before } \\
\text { improved }\end{array}$ & 23.7 & 26.7 & 78.41 & 5 & 7591 \\
\hline $\begin{array}{c}\text { After } \\
\text { improved }\end{array}$ & 21.1 & 19.4 & 55.21 & 0 & 7896 \\
\hline
\end{tabular}

As shown in the table above, the average vehicle delay of simulations status is $23.7 \mathrm{~s}$ which has little difference from the delay of $24.8 \mathrm{~s}$ obtained by status survey, therefore, the Vissim simulation 
model is effective.

\section{Conclusion}

The paper targets at minimize conflicts between pedestrian and vehicle, mixes traffic status of road intersections and designs the signal phase optimization scheme based on pedestrian first. Moreover, it conducts comparison evaluation after simulating actual urban road intersections. The result shows that the optimization scheme can reduce conflicts between pedestrian and vehicle, decrease the vehicle parking rate, increase space-time resources during pedestrian crossing and optimize the service level of pedestrian crossing at intersections.

\section{References}

[1] X.G.Yang :The Shanghai public security traffic management planning[R]. Shanghai, 2002.

[2] L.Zhao, C.Q.Shao, L.Wen: Journal of Road Traffic \& Safety Vol.8(2008), p.27-30.

[3]Y.Ni, K.P.L: Journal of Transport Information and Safety Vol.8(2007),p.22-26.

[4] W.J.Ma, Y.Lin, X.G.Yang: Journal of Traffic and Transportation Engineering Vol.4(2004),p. 103-106.

[5] X.G.Yang, W.J.Ma, Y.Lin: Highway Traffic Science and Technology Vol.22(2005),p. 127-131.

[6] C.F.Shao, X.M.Chen: Traffic and Transportation System Engineering and Information Vol.7(2007),p. 18-23. 